\title{
The design and investigation of room temperature thermotropic nematic gold nanoparticles
}

\author{
L.Cseh, G. H. Mehl
}

\section{Supporting information}

\begin{abstract}
Instrumentation
Nuclear magnetic resonance (NMR) spectra were taken on a Jeol JNM-ECP $400 \mathrm{MHz}$ FT-NMR spectrometer. Chemical shifts are reported in ppm relative to TMS. The thermal properties were investigated using a Mettler Toledo differential calorimeter (DSC) $822^{\mathrm{e}}$ in nitrogen against an indium standard. Transition temperatures were determined as the onset of the maximum in the endotherm or exotherm. The mesophases were studied on an Olympus BH-2 optical polarising microscope, equipped with a Mettler FP82 HT hot stage and a Mettler FP90 central processor. Pictures of the mesophases were taken using a JVC digital video camera connected to a PC. Software Studio Capture, supplied by Studio86Designs was used for image capturing. Transmission electron micrographs were recorded with a JEOL JEM 3010 Transmission Electron Microscope (point resolution $0.17 \mathrm{~nm}$ ).equipped with a GATAN GIF 200 electron imaging filter.
\end{abstract}

NMR Spectra (solvent $\mathrm{CDCl}_{3:}$ a) 1; b), hexylthiol substituted nanoparticle system; c) N1H. 
a)

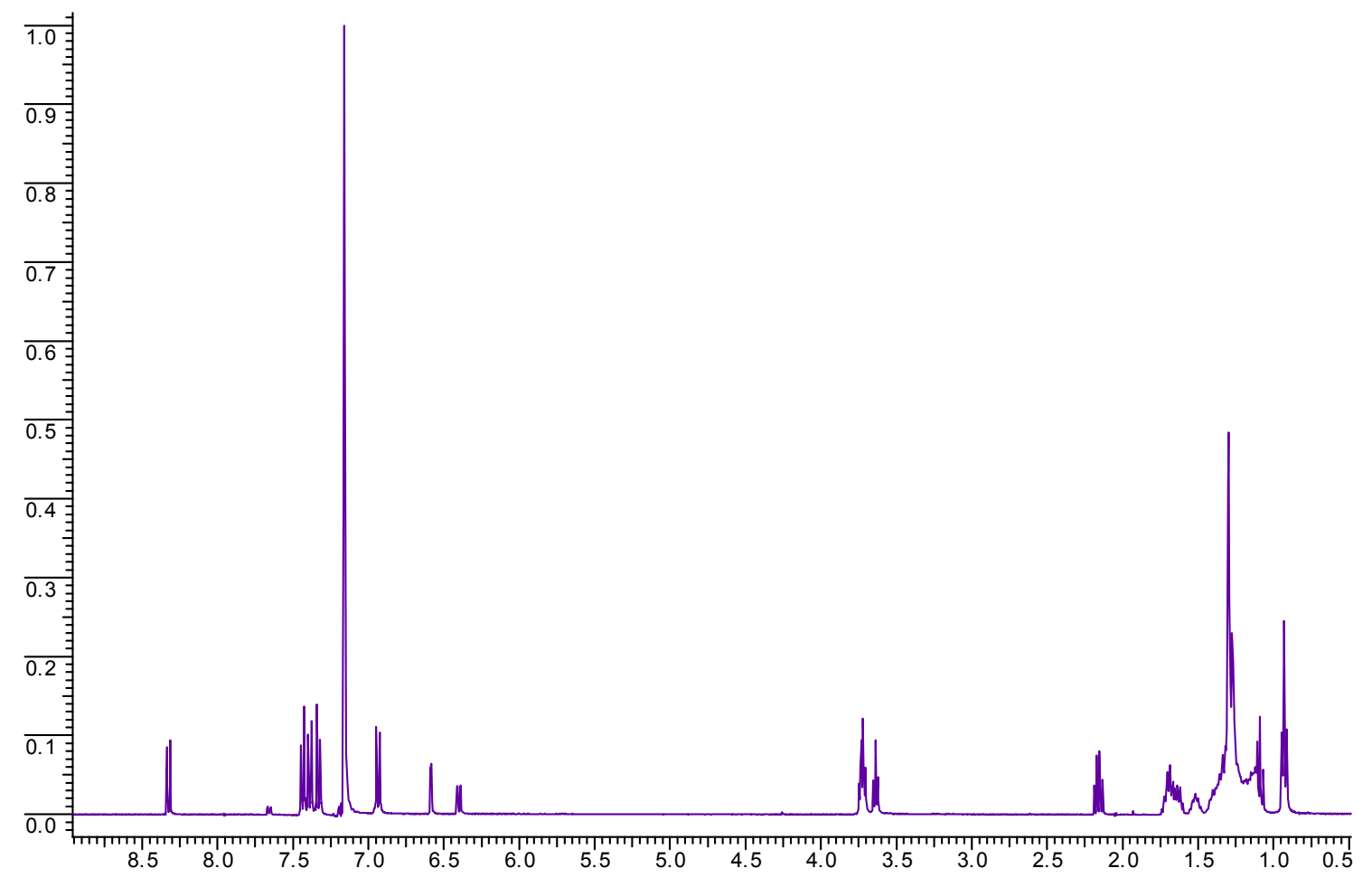


b)

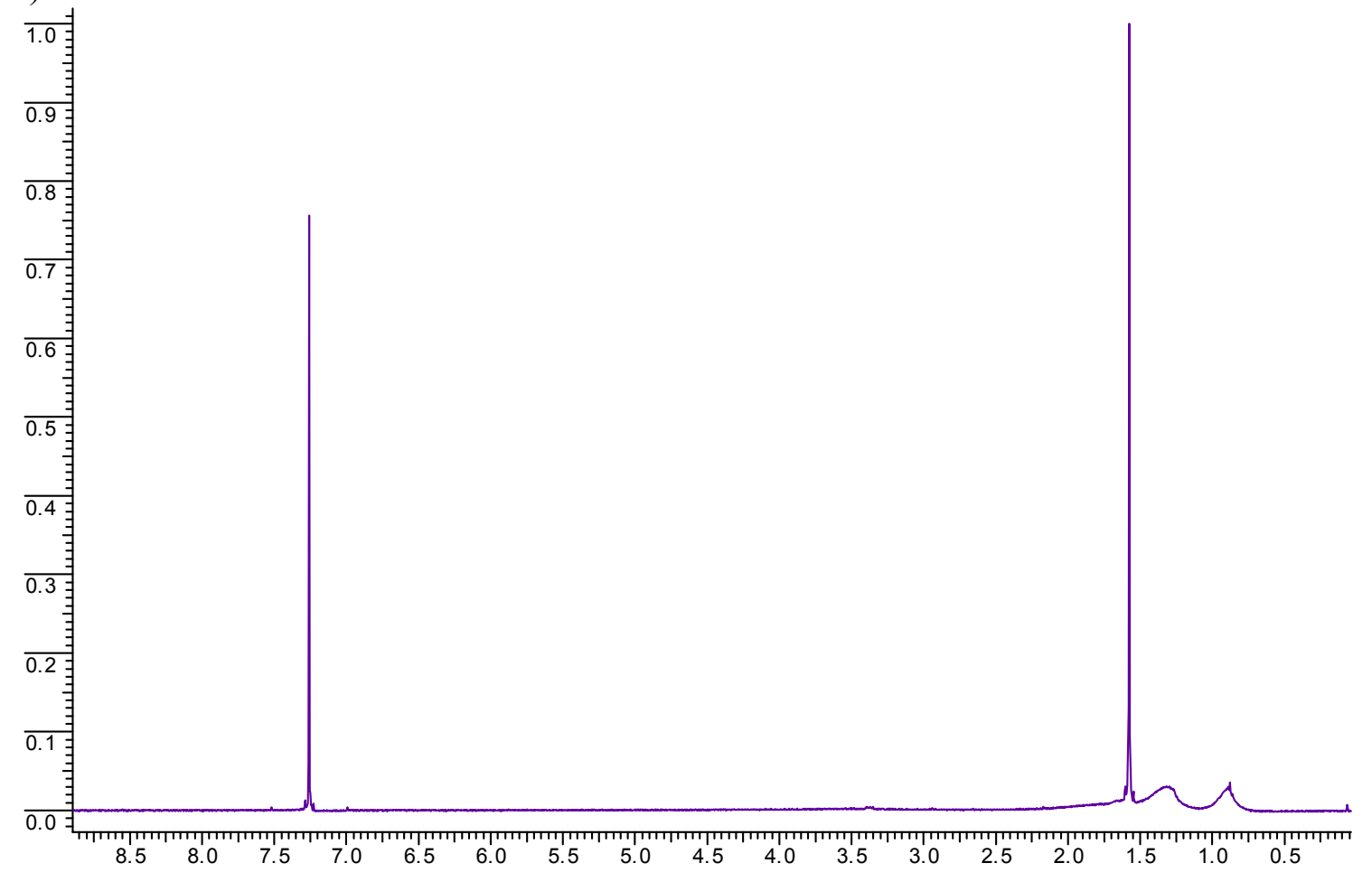

c)

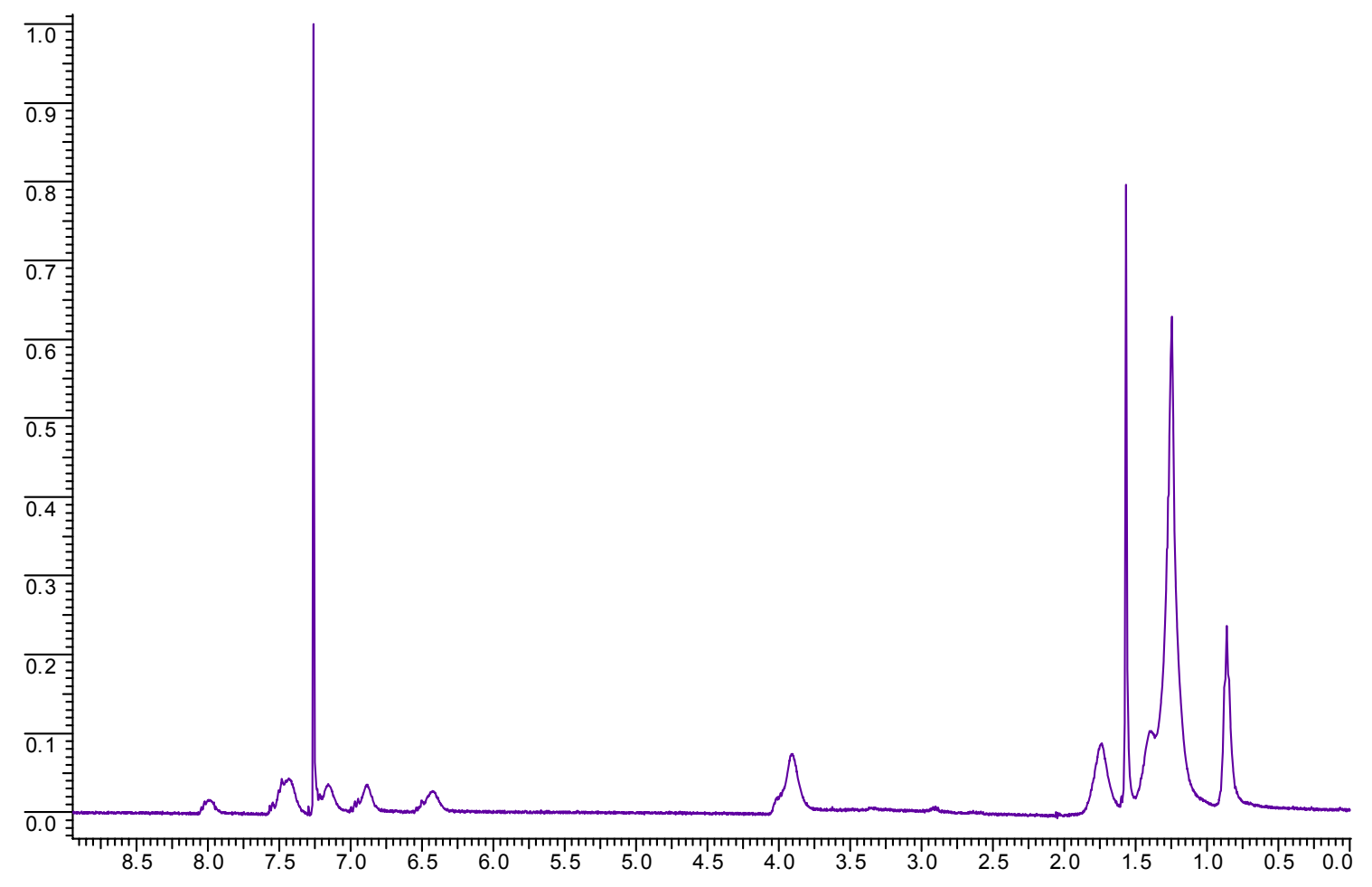


DSC trace of N1H. The glass transition has a very low $\Delta \mathrm{Cp}$ value, and was therefore not analyzed further.

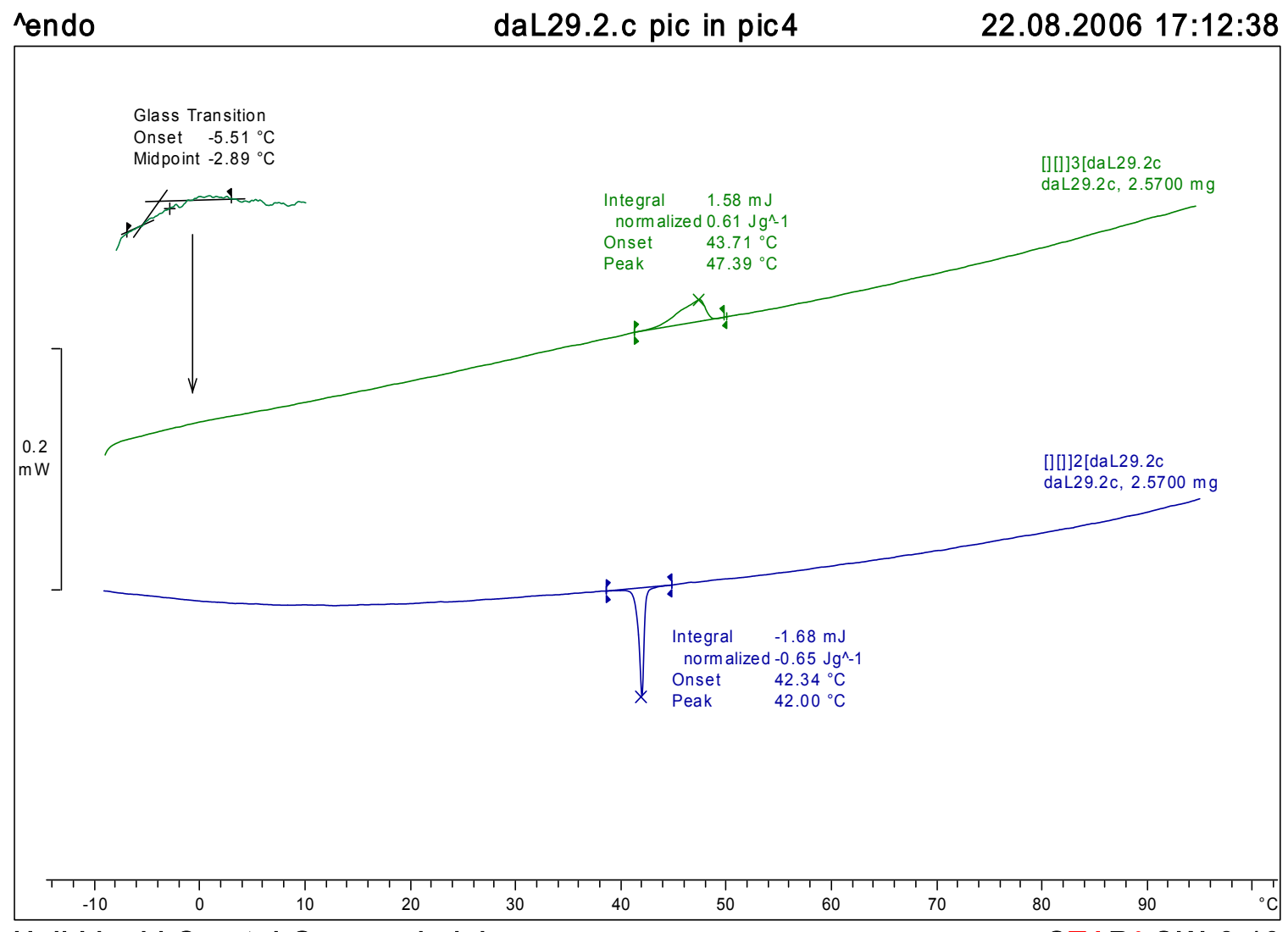

Hull Liquid Crystal Group: chsjah $\quad$ STAR $^{\mathrm{e}}$ SW 8.10

TEM picture of $\mathbf{N 1 H}$ on a graphite surface. Scale bar $=5 \mathrm{~nm}$

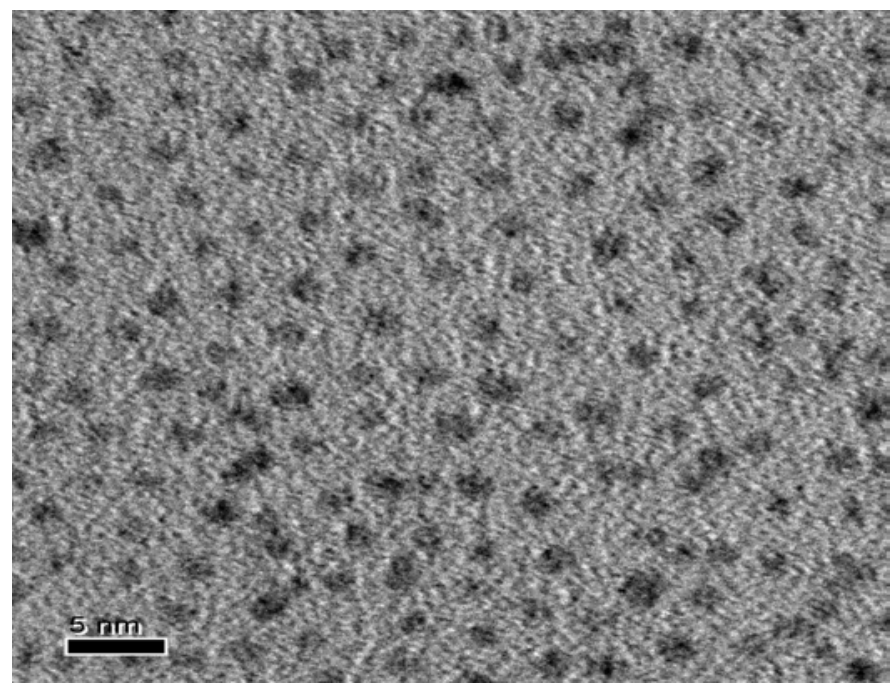




\section{Calculation of the of the number of organic groups covering the surface of the napoarticles}

The number of gold atoms/particle was calculated using the following formula [1, 2]:

$$
\mathrm{N}_{\mathrm{Au}}=\frac{4 * \pi * R^{3}}{3 * v_{g}}=\frac{4 * \pi * D^{3}}{8 * 51}
$$

where: $\mathrm{R}$ - radius of nanoparticle $(\AA)$; D - diameter of nanoparticule $(\AA) ; v_{\mathrm{g}}$ - volume of gold atom $\left(v_{\mathrm{g}}=17 \AA^{3}\right)$.

The diameter of nanoparticles was determined by transmission electron microscopy (TEM). The dimension of nanoparticles was determined to be $1.6 \pm 0.4 \mathrm{~nm}$. Each particle contains about 140 gold atoms/ particle.

The ${ }^{1} \mathrm{H}-\mathrm{NMR}$ spectra were used to prove the purity of nanoparticles and to calculate the ratio of alkaneanthiol to mesogens $\mathbf{1}$ attached to a particle.

\section{Synthesis of 1}

The compound 4'-(undecyloxy)biphenyl-4-yl 2-(11-mercaptoundecyloxy)-4-(octyloxy) benzoate (1) was obtained in two steps. The first step is free-radical addition reaction induced by AIBN as initiator [3] to the starting material $\mathbf{A}$ [4] and the second step is a mild deprotection reaction, which was carried out using TFA at room temperature. The thiol was isolated by column chromatography $\left(\mathrm{SiO}_{2}, \mathrm{CH}_{2} \mathrm{Cl}_{2} /\right.$ hexane $\left.=6 / 4, \mathrm{Rf}=0.43\right)$. Recrystallization from hexane yielded a white solid product. 


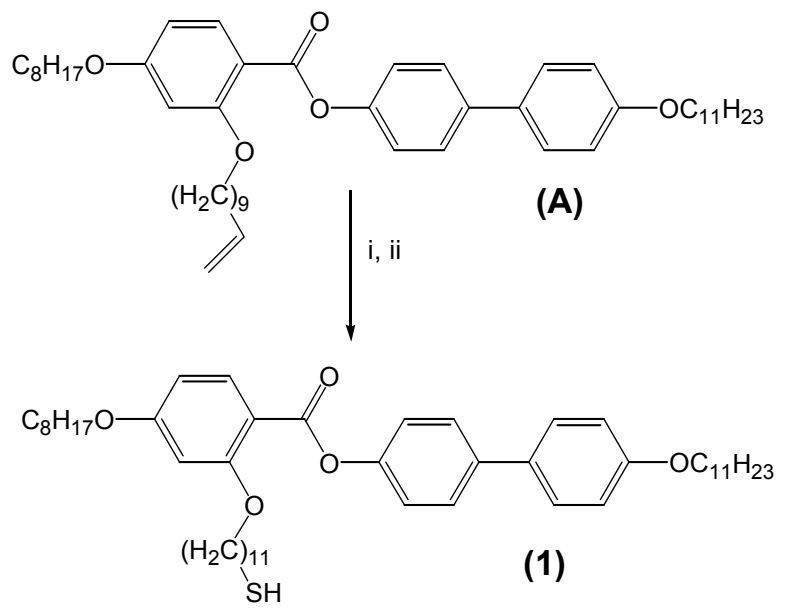

Scheme 1. Reagents and conditions: i), $\mathrm{Ph}_{3} \mathrm{SiSH}$ (1.4 eq), AIBN, benzene, reflux 38h; ii), TFA (5 eq), room temperature, $30 \mathrm{~min}$.

(1) Yield: 34\%; elemental analysis calc. for $\mathrm{C}_{49} \mathrm{H}_{74} \mathrm{O}_{5} \mathrm{~S}, \mathrm{C}, 75.92 ; \mathrm{H}, 9.62 ; \mathrm{S}, 4.14$. Found: C, 75.80; H, 9.50; S, 4.19\%;

${ }^{1} \mathrm{H}-\mathrm{NMR}\left(400 \mathrm{MHz}\right.$ in $\left.\mathrm{C}_{6} \mathrm{D}_{6}\right) \delta[\mathrm{ppm}]: 0.92\left(\mathrm{t}, 6 \mathrm{H},-\mathrm{CH}_{3}\right), 1.08(\mathrm{t}, 1 \mathrm{H},-\mathrm{SH}), 1.10-1.55(\mathrm{~m}$, $\left.42 \mathrm{H},-\mathrm{CH}_{2}-\right), 1.66\left(\mathrm{~m}, 6 \mathrm{H},-\mathrm{CH}_{2}-\right), 2.15\left(\mathrm{dt}, 2 \mathrm{H},-\mathrm{CH}_{2}-\mathrm{SH}\right), 3.62\left(\mathrm{t}, 2 \mathrm{H},-\mathrm{O}-\mathrm{CH}_{2}-\right), 3.71(\mathrm{~m}$, $\left.4 \mathrm{H},-\mathrm{O}-\mathrm{CH}_{2}-\right), 6.39\left(\mathrm{~d}, 1 \mathrm{H}, \mathrm{H}_{\mathrm{ar}}\right), 6.57\left(\mathrm{dd}, 1 \mathrm{H}, \mathrm{H}_{\mathrm{ar}}\right), 6.93\left(\mathrm{~m}, 2 \mathrm{H}, \mathrm{H}_{\mathrm{ar}}\right), 7.32\left(\mathrm{~m}, 2 \mathrm{H}, \mathrm{H}_{\mathrm{ar}}\right)$, 7.38(m, $\left.2 \mathrm{H}, \mathrm{H}_{\mathrm{ar}}\right), 7.42\left(\mathrm{~m}, 2 \mathrm{H}, \mathrm{H}_{\mathrm{ar}}\right), 8.31\left(\mathrm{~d}, 1 \mathrm{H}, \mathrm{H}_{\mathrm{ar} .}\right)$;

${ }^{1} \mathbf{H}-\mathrm{NMR}$ of NH1: $\left(400 \mathrm{MHz}\right.$ in $\left.\mathrm{CDCl}_{3}\right) \delta[\mathrm{ppm}]: 0.86\left(-\mathrm{CH}_{3}\right), 1.24\left(-\mathrm{CH}_{2}-\right), 1.74\left(-\mathrm{CH}_{2}-\right)$, 3.88(-O- $\left.\mathrm{CH}_{2}-\right), 6.41\left(\mathrm{H}_{\mathrm{ar}}\right), 6.86\left(\mathrm{H}_{\mathrm{ar}}\right), 7.13\left(\mathrm{H}_{\mathrm{ar}}\right), 7.43\left(\mathrm{H}_{\mathrm{ar}}\right), 8.02\left(\mathrm{H}_{\mathrm{ar}}\right)$.

The results on nanoparticles partially covered with LC groups, not showing liquid crystalline behavior in the bulk have been reported as a poster presentation in: L.Cseh, G.H Mehl, Poster COL-P056, Book of Abstracts, 20 ${ }^{\text {th }}$ Int. Liquid Crystal Conf, 04-09. 07. 2004, Ljubljana, Slovenja.

[1] Daniel, M. C. ; Ruiz, J.; Nlate, S.; Blais, J. C. ; Astruc, D.; J. Am. Chem. Soc., 2003, 125, 2617;

[2] Leff, D. V.; Ohara, P. C.; Heath, J. R.; Gelbart, W. M.; J. Phys. Chem., 1995, 99, 7036;

[3] Haché, B.; Gareau, Y.; Tetrahedron Lett., 1994, 35(12), 1837;

[4] Diez, S.; Dunmur, D.A. ; De la Fuente, M. R. ; Karahaliou, P. K.; Mehl, G. H.; Meyer, T.; Jubindo, M. A. P.; Photinos, D. J.; Liq. Cryst. 2003, 30, 1021. 